\title{
Antenatal care visits of child-bearing mothers having a child five years preceding in the survey and associated factors in Benchi-Sheko Zone, Southwestern Ethiopia:Cross-sectional study
}

\section{Assaye Belay Gelaw ( $\sim$ abstat23@gmail.com )}

Mizan-Teferi Teachers College: Mizan-Tepi University https://orcid.org/0000-0001-7315-9141

\section{Solomon Abebaw Andargie}

Mizan-Tepi University

Abiyot Negsh Terefe

Jimma University

\section{Research}

Keywords: ANC visit, Odd ratio, Poisson Regression Model, Benchi-Sheko Zone, Women

Posted Date: February 17th, 2021

DOI: https://doi.org/10.21203/rs.3.rs-167661/v3

License: (c) (1) This work is licensed under a Creative Commons Attribution 4.0 International License.

Read Full License 
Antenatal care visits of child-bearing mothers having a child five years preceding in the survey and associated factors in Benchi-Sheko Zone, Southwestern Ethiopia:Cross-sectional study Assaye Belay Gelaw ${ }^{1 *}$,Solomon Abebaw Andargie ${ }^{2}$, Abiyot Negash Terefe ${ }^{3}$

$1^{*, 2}$ lecturer of statistics ,Mizan-Tepi University, Ethiopia.

${ }^{3}$ lecturer of statistics, Jimma University, Ethiopia.

\section{1* Corresponding author:Assaye Belay Gelaw}

E-mail:abstat23@gmail.com

\section{Abstract}

Background: Antenatal care (ANC) is the service given by the caregiver for pregnant women to make safe the health of both pregnant women and babies during the pregnancy period. The objective of the current study was to persuade high-quality public health service and plan the mothers and their households expressively and mentally for being motherhood by everincreasing antenatal care visits from health institutions.

Methods: Community-based cross-sectional study design was applied. Cross-sectional survey design mainly used for the collection of information on the Utilization of Antenatal Care Visit of Women During Pregnancy and its Related Factors in Bench-Sheko Zone, South Nation Nationalities Peoples of Region, Southwestern Ethiopia: Cross-sectional study. The study populations are all populations who are living in the study area, South Nation Nationalities peoples of Region, Southwestern Ethiopia.

Results: Evidence tells us, one hundred twenty-nine (16.9\%) of pregnant women were not visited by the caregiver during pregnancy. Around eighty-three percent of pregnant women were visited in health institutions at least once prior in the five years in the survey. The Poisson regression model was preferred to fit the data. As the output indicated in analysis, the odds ratio of women whose husband education status is illiterate is equal to $\exp (-0.272)=0.76(95 \%$ CI:$0.507,-0.038$ ) (other variables are adjusted), it indicates that the women whose husband education status is 0.76 less likely to ANC visit than women whose husband education status is higher and above.

Conclusions: The remark conclusion that the source of information, religion, educational status, birth order, knowledge of danger signs for pregnancy, and service satisfaction were significant at the alpha level of significance on the ANC visit of Women during pregnancy.

Keywords: ANC visit; Odd ratio; Poisson Regression Model; Benchi-Sheko Zone;Women 


\section{Plain English Summary delight}

Antenatal care is among the maternal care services provided to pregnant women to reduce pregnancy complications. The authors of this study aimed to assess the antenatal care visit utilization of child-bearing mothers having a child five years preceding in the survey and associated factors in Bench-Sheko Zone.

The questionnaires were prepared and validated by researchers for collecting in formations from participants to assess the number of antenatal care visits of child-bearing mothers. The pregnancy complication will be reduced if the number of antenatal care visits in health service is increased. According to the world health organization (WHO), any healthy pregnant women attend at least four number of ANC visits. However, women with health complications are recommended for more ANC visits in health institutions.

The aim of this study was to evaluate how many ANC visits in health institutions during the pregnancy period.

Out of 764 participants, $83.1 \%$ had attended ANC visit at least one in health institutions, and $16.9 \%$ of participants had not attended any health institutions.

In conclusion, the ANC visit of child-bearing mothers during pregnancy in the study found lower, and this information can be used as input for both policymakers and health professionals to design a better way in providing ANC visits and improve maternal health care. 


\section{Background}

Antenatal care (ANC) is the service given by the caregiver for pregnant women to make safe the health of both pregnant women and babies during the pregnancy period [1]. A significant antenatal care visit of pregnant women is a significant incident to persuade high-quality health outcomes and plan women on their households meaningfully and mentally for being motherhood by ever-increasing antennal care visits from either any clinics or hospitals [2]. Ninety-eight percent of pregnant mothers had received antenatal care visits at least one in developed countries, and sixty-eight percent of women had attended ANC visits in developing countries. [3]. A deficiency in pertinent ANC visits is the main issue in sub-Saharan Africa [4].

In Sub- Saharan Africa including Ethiopia, most pregnant mothers did not start to attend ANC visits in the first trimester even if most pregnancy history screen out in this stage, however, they took antenatal care visit at the last two trimesters( two and second trimesters ) [5]. Some factors may be hinder not taking ANC visits in the first trimesters like the education status of women and family [6], and the most important demographic factor and age are very critical for any issue regarding pregnancy complication [7].

In many work of literature, and according to the World Health Organization (WHO) recommendation, pregnant women should attend ANC visit in a minimum of four times during pregnancy until giving for a healthy pregnancy [8]. The number of ANC visits may decrease or increase depending upon the health status of women as well as the prevalence of risks. Hence, healthy pregnant women will attend ANC visits three times, two times and one time. In this regard, the dependent variable (count) measured in count form, and the model ( Poisson regression model ) should be mandatory to make sound or a representative decision to avoid misleading conclusions and recommendations[9].

The previous study was done in Sheka, Bench-Maji, and Kaffa zones showed that the prevalence of childbearing mothers attend ANC visit service utilization is very high compare to women those never ANC visit utilized in their last pregnancy period [9]. An antenatal care visit is the most anticipation strategy that makes it possible to reduce women and baby mortality. Communicable and no communicable diseases expected to be managed and identified during 
ANC visit utilization [10]. Thus, the study was aimed to determine the number of ANC visit utilization women during pregnacey period in Bench-Sheko zone, Southwestern Ethiopia.

\section{Methods}

The data collected by the data collectors from Bench-Sheko Zone's kebeles. Bench- Maji Zone now it is called Bench-Sheko Zone. Bench-Sheko Zone is one of the zones in South, Nation, Nationality Peoples of Region. It has mount Guraferda and Omo National park. The main crops are Maize, Godere, Enset, Sorghum, spices; different fruits, coffee, and honey are common in the zone[11]. The sample size calculated using a single population proportion formula based on the following assumptions: Forty-four of the respondents started their ANC within the recommended time, ninety-five percent of the confidence interval, five percent of margin of error, and design effect considered, and seven hundred sixty-four participants have participated.

\section{Study Variables}

The dependent variable is antenatal care visits of child-bearing mothers having a child five years preceding in the survey. Independent variables are as follows: residents, mother's level of education, husband's level of education, Marital status, Religion, distance in a kilometer, service satisfactory, birth order, mother's occupation, husband's occupation, age of mothers, mother's Knowledge of danger sign of pregnancy complication, the problem of transportation, information source, and mother's age at first pregnancy. The dependent variable is antenatal care visits of child-bearing mothers having a child five years preceding in the survey.

\section{Statistical Methods}

Descriptive statistics were computed to the characteristics of the respondents using percentage, mean, and standard deviation.

Poisson Regression Model is the model that fits the count response that follows the Poisson distribution. The value of the dependent variable is counted as $0,1,2 \ldots \ldots \ldots .$. and the assumption of a model is equality of mean and variance(variance greater than mean). The negative binomial regression model allows overdispersion that accounts for unobserved heterogeneity that comes from observation i[12]. Thus, Poisson and Negative binomial regression modes can be 
employed to determine the significant contribution of factors on ANC visits of women during pregnancy in the Benchi-Sheko Zone, Ethiopia.

The poisson density function is given by : $p(Y=y)=\frac{e^{-\lambda} \lambda^{y}}{y !}, \mathrm{y}=0,1,2,3, \ldots(1)$

Where, $\lambda$ is parameter, $\mathrm{y}$ is varaiable. $E(Y=y)=\operatorname{var}((Y=y) \ldots \ldots \ldots \ldots \ldots . .(1.1)$

Let $x_{i}, \lambda_{i} \beta$ are $\mathrm{k}$ - independent varaiables, rate or mean and parameter respectively. Exponetialy expressed in poisson regression: $\left.\lambda_{i}=e^{\left(x_{i}{ }^{i} \beta\right)}, i=1,2,3, \ldots \ldots, n \ldots \ldots \ldots . . .2\right)$

Given (1) and (1.2) and the assumption that the observations $y_{i} / x_{i}$ are independent, the most natural estimator is maximum likelihood (ML). The log-likelihood function is

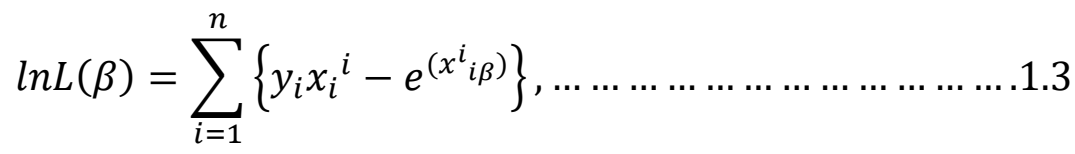

The Poisson MLE, denoted $\beta_{i}$, is the solution to $\mathrm{k}$ nonlinear equations corresponding to the First-order condition for maximum likelihood,

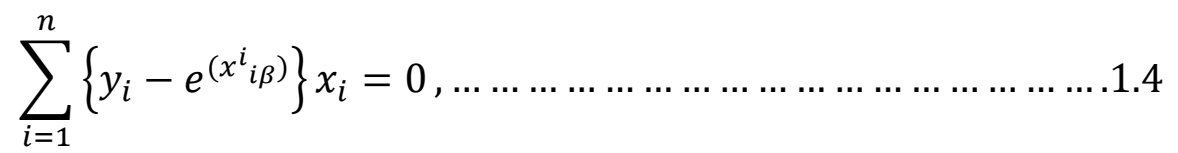

By the standard maximum likelihood theory of correctly specified models, the estimator $\hat{\beta}_{p}$ is consistent for $\beta$ and asymptotically normal with the sample covariance matrix [9].

\section{Negative Binomial Regression Model}

The negative binomial regression model was of the form [13];

$Y_{i}=0,1,2, \ldots \ldots$. with the probability $\frac{\Gamma\left(\frac{1}{\alpha}+y_{i}\right)}{\Gamma\left(\frac{1}{\alpha}\right)\left(y_{i}+1\right)}\left(\frac{1}{1+\alpha * \lambda_{i}}\right)^{\frac{1}{\alpha}}\left(\left(\frac{\alpha * \lambda_{i}}{1+\alpha * \lambda_{i}}\right)^{\mathrm{y}_{\mathrm{i}}} \ldots \ldots \ldots \ldots \ldots . . . . . .5\right.$

$\lambda_{i}=e^{\beta_{i} x_{i}}$

Where $x_{i}$ is the $\mathrm{i}^{\mathrm{t}}$ covariate and $\beta_{i}$ Is the regression coefficient of log it model.

\section{Model Selection Criteria}

Akaike Information Criterion (AIC) and the Basic information criterion (BIC) used to judge the performance of the model. Smaller the AIC and BIC value, the best model. [14].

\section{Parametric Estimation}

The parameter estimators were estimated by maximum likelihood function and obtained by partial derivatives equal to zero [15]. 


\section{Model Comparison Criterion}

The different models can be compared as they were estimated by likelihood fuanction. And its pereformace of different models can be compared by Akkakian and Bayesian information criteria. The smallest akkkaian and bayesisn information is the better one . It is given by $A I C=$ $-2 l+2 p$, where,$l$ denotes the $\log$ likelihood evaluated for estimated parameters $\lambda$ and $p$ For this measure, the smaller the AIC, the better the model [16].

\section{Results}

Seven hundred sixty-four mothers had given birth within five years before the survey. Table 1 shows that the women residing in rural residents (50.8\%) and urban area (49.2\%); women with single $(0.3 \%)$, women with married $(84.6 \%)$, women with divorced(10.6\%), women with widowed (4.2\%), women with Orthodox(38.9\%), women with Catholics (3.4\%), women with Muslim(15.6\%), women with protestant(38.2\%) and women with tradition (3.9\%) had attended at health institutions during the pregnancy period.

Concerning information source, women who gained information about the antenatal care visit from radio service, health workers, traditional delivers, relatives, partners, and others are seven $(0.6 \%)$, six hundred eighty-seven $(97.7 \%)$, thirteen $(0.2 \%)$, eleven $(0.4 \%)$, and one $(0.1 \%)$ respectively had attended antenatal care visits service from nearest health institutions.

Table1.Summary of Socio-demographic Factors on the Average Antenatal Care Visit Utilization of Women in Benchi-Sheko Zone

\begin{tabular}{lccccc}
\hline Variables & Categories & $\mathrm{N}$ & $\%$ & Average & Std.deviation \\
\hline \multirow{3}{*}{ Residence } & & & & & \\
& Urban & 461 & 50.8 & 2.99 & 2.267 \\
& Rural & 303 & 49.2 & 4.11 & 1.071 \\
& Single & 3 & 0.3 & 2.67 & 3.055 \\
Marital status & Married & 643 & 84.6 & 3.33 & 2.044 \\
& Divorced & 79 & 10.9 & 3.49 & 1.376 \\
& Windowed & 39 & 4.2 & 2.72 & 2.051 \\
Religions & Orthodox & 276 & 38.9 & 3.57 & 1.862 \\
& Catholic & 22 & 3.4 & 3.95 & 1.253 \\
& Muslim & 105 & 15.6 & 3.76 & 1.644 \\
& Protestant & 296 & 38.2 & 3.27 & 2.101 \\
& Traditional & 65 & 3.9 & 1.51 & 1.751 \\
Mother's & Illiterate & 329 & 32.0 & 2.46 & 2.113
\end{tabular}




\begin{tabular}{|c|c|c|c|c|c|}
\hline \multirow[t]{2}{*}{ education } & Elementary & 201 & 28.4 & 3.58 & 2.014 \\
\hline & Secondary & 102 & 17.6 & 4.37 & 1.177 \\
\hline \multirow{4}{*}{$\begin{array}{l}\text { Husband's } \\
\text { education }\end{array}$} & Higher + & 132 & 25.9 & 4.2 & 1.032 \\
\hline & Illiterate & 255 & 24.3 & 2.42 & 2.115 \\
\hline & Elementary & 184 & 23.5 & 3.23 & 2.277 \\
\hline & Secondary & 104 & 14.8 & 3.61 & 1.451 \\
\hline \multirow{4}{*}{$\begin{array}{c}\text { Mother's } \\
\text { occupation }\end{array}$} & Higher+ & 221 & 37.4 & 4.28 & 1.173 \\
\hline & Farmer & 462 & 50 & 2.74 & 2.225 \\
\hline & Trade & 136 & 22.2 & 4.13 & 1.198 \\
\hline & Piecework & 4 & 0.8 & 4.75 & 1.500 \\
\hline \multirow{4}{*}{$\begin{array}{l}\text { Husband's } \\
\text { occupation }\end{array}$} & Office & 162 & 27 & 4.22 & 0.991 \\
\hline & Farmer & 424 & 44.8 & 2.67 & 2.200 \\
\hline & Trade & 101 & 15.9 & 3.99 & 1.539 \\
\hline & Piecework & 5 & 9.7 & 3.6 & 0.894 \\
\hline \multirow{3}{*}{ Abortion status } & Office & 234 & 38.6 & 4.17 & 1.221 \\
\hline & Yes & 74 & 10.4 & 3.57 & 1.580 \\
\hline & No & 690 & 89.6 & 3.29 & 2.030 \\
\hline \multirow[t]{4}{*}{ Decision making } & Myself & 78 & 10.7 & 3.46 & 1.365 \\
\hline & Husband & 15 & 0.7 & 1.20 & 1.568 \\
\hline & Jointly & 668 & 88.1 & 3.34 & 2.027 \\
\hline & Others & 3 & 0.6 & 4.67 & 4.041 \\
\hline
\end{tabular}

Table 1. (Continued ...). Summary of Socio-demographic Factors on the Average Antenatal Care Visit Utilization of Women in Benchi -Sheko Zone.

\begin{tabular}{cccccc}
\hline Variables & Categories & $\mathrm{N}$ & $\%$ & Average & Std.deviation \\
& & & & & \\
\hline \multirow{2}{*}{$\begin{array}{c}\text { Knowledge about } \\
\text { danger sign }\end{array}$} & Yes & 3454 & 77.8 & 4.33 & 1.300 \\
Birth order & No & 310 & 22.2 & 1.83 & 1.880 \\
& {$[1,2)$} & 111 & 15.1 & 3.45 & 2.092 \\
& {$[2,3)$} & 315 & 30.2 & 2.43 & 2.002 \\
Source of & {$[3,5)$} & 236 & 36.3 & 3.90 & 1.629 \\
information & $5+$ & 102 & 18.3 & 4.55 & 1.317 \\
& Radio & 7 & 0.6 & 2 & 2.160 \\
& Health worker & 687 & 97.7 & 3.6 & 1.811 \\
& Traditional delivers & 13 & 0.2 & 0.38 & 1.121 \\
& Relatives & 11 & 0.4 & 1 & 2.683 \\
& Partners & 1 & 0.1 & 2 & ---- \\
& Others & 45 & 1 & 0.58 & 1.390
\end{tabular}




\begin{tabular}{cccccc} 
A transportation & Yes & 64 & 9.5 & 3.75 & 1.491 \\
problem & No & 700 & 90.5 & 3.27 & 2.202 \\
Service & Yes & 540 & 85.7 & 4.02 & 1.508 \\
satisfaction & No & 224 & 14.3 & 1.61 & 1.990 \\
Age of mothers & $15-21$ & 4 & 0.6 & 3.5 & 1.291 \\
& $22-28$ & 125 & 13.7 & 2.77 & 1.931 \\
& $29-35$ & 284 & 39.5 & 3.52 & 1.852 \\
Age of first & $36-42$ & 171 & 24.1 & 3.57 & 1.955 \\
pregnancy & $43-49$ & 180 & 22.2 & 3.12 & 2.205 \\
& $15-21$ & 87 & 8.3 & 2.43 & 1.933 \\
& $22-28$ & 270 & 32.8 & 3.07 & 2.035 \\
Distance & $29-35$ & 212 & 31.5 & 3.76 & 1.753 \\
& $36-42$ & 129 & 18.3 & 3.60 & 1.707 \\
& $43-49$ & 66 & 9 & 3.47 & 2.573 \\
& $<=$ Five km & 337 & 52 & 3.91 & 1.771 \\
& $>$ Five km & 427 & 48 & 2.84 & 2.032 \\
\hline
\end{tabular}

Table 1 shows that the percentage and an average number of antenatal care visit of women whose current jobs such as farmers, trade, piece work, and office are four hundred sixty-two (50\%), one hundred thirty-six (22.2\%), four(0.8\%), two hundred thirty-four (38.6\%), and 2.74,4.13,4.75 and 4.17 in average utilized antenatal care visit utilization of childbearing mothers from nearby health sectors. Furthermore, there is less variation among women whose job status is office.

Women's religion and marital status had a significant effect on ANC visits utilization. Women whose marital status are single, married, divorced, windows are three $(0.3 \%)$, six hundred fortythree $(84.6 \%)$, seventy-nine $(10.9 \%)$, thirty-nine $(4.2 \%)$, and 2.67,3.33,3.49,3.49 and 2.72 attended ANC visits in a health institution on average. About religion, women whose religious are orthodox, catholic, Muslim protestant and tradition are two hundred seventy-six (38.6\%), twenty-two (3.4\%), one hundred five ((15.6\%), two hundred ninety-six (38.2\%), and 3.57, 3.95,3.76 and 3.27 utilized in average. Besides, the standard deviation showed that there is no such difference among women for ANC visits.

Table 2. Summary of Socio-demographic Factors on the Average Antenatal Care Visit Utilization of Women in Benchi -Sheko Zone 


\begin{tabular}{lllllllllll}
\hline Number of visit & 0 & 1 & 2 & 3 & 4 & 5 & 6 & 7 & 8 & 9 \\
\hline Frequency & 129 & 48 & 44 & 55 & 339 & 63 & 59 & 14 & 10 & 3 \\
Percentage & 16.9 & 6.3 & 5.8 & 7.2 & 44.4 & 8.2 & 7.7 & 1.8 & 1.3 & 0.4 \\
Comul.percentage & 16.9 & 23.2 & 28.9 & 36.1 & 80.5 & 88.7 & 96.5 & 98.3 & 96.6 & 100 \\
\hline
\end{tabular}

Table 2 shows that women did not attend ANC visits during pregnancy. Evidence tells us, one hundred twenty-nine (16.9\%) did not take ANC visits at the health institution. Thirty-six women attended bellow four ANC visits during pregnancy. It is below a minimum of world health organization recommendation.

Table 3. Model Comparision

\begin{tabular}{lccc}
\hline Model type & Link function & Log Likelihood & $\begin{array}{l}\text { Akaike's Information } \\
\text { Criterion (AIC) }\end{array}$ \\
\hline Poison Regression model & Log & -1325.289 & 2740.579 \\
Negative Binomial Model & Log & -1675.436 & 3440.871 \\
\hline
\end{tabular}

The smallest AIC and BIC value is preferable. Thus, the output shown in table 3 showed that the Poisson regression model is responsible for fitting the data. There fore, using the forward variable selection method of step by step, the model is given in table 4 was illustrated..

The odds ratio for women who had lived in urban resident equals to $\exp (0.226)=1.25(95 \% \mathrm{CI}$ : 0.027,0.426) (other variables are adjusted). It indicated that those women who had lived in urban residents are 1.25 times more likely to attend health institutions than women who had lived in rural residents (Table 4). The odd ratio of women whose religion is Catholic is equal to $\exp (0.093)=1.46(95 \% \mathrm{CI}: 0.067,0.691)$ (other variables are adjusted). It implies that the women whose religion is catholic are 1.46 times more likely to have more visits to the health center than women whose religion was traditional. To some extent, husband education status is a significant effect on the women to take ANC visit utilization. As the output indicated in table 4, the odds ratio of women whose husband education status is illiterate is equal to $\exp (-0.272)=0.76(95 \%$ CI:-0.507,-0.038) (other variables adjusted), it indicates that the women whose husband education status is 0.76 less likely to visit ANC visit than women whose husband education status is higher and above. 
Table 4:Maximum likelihood Parameter Estimates of Poisson Regression Model

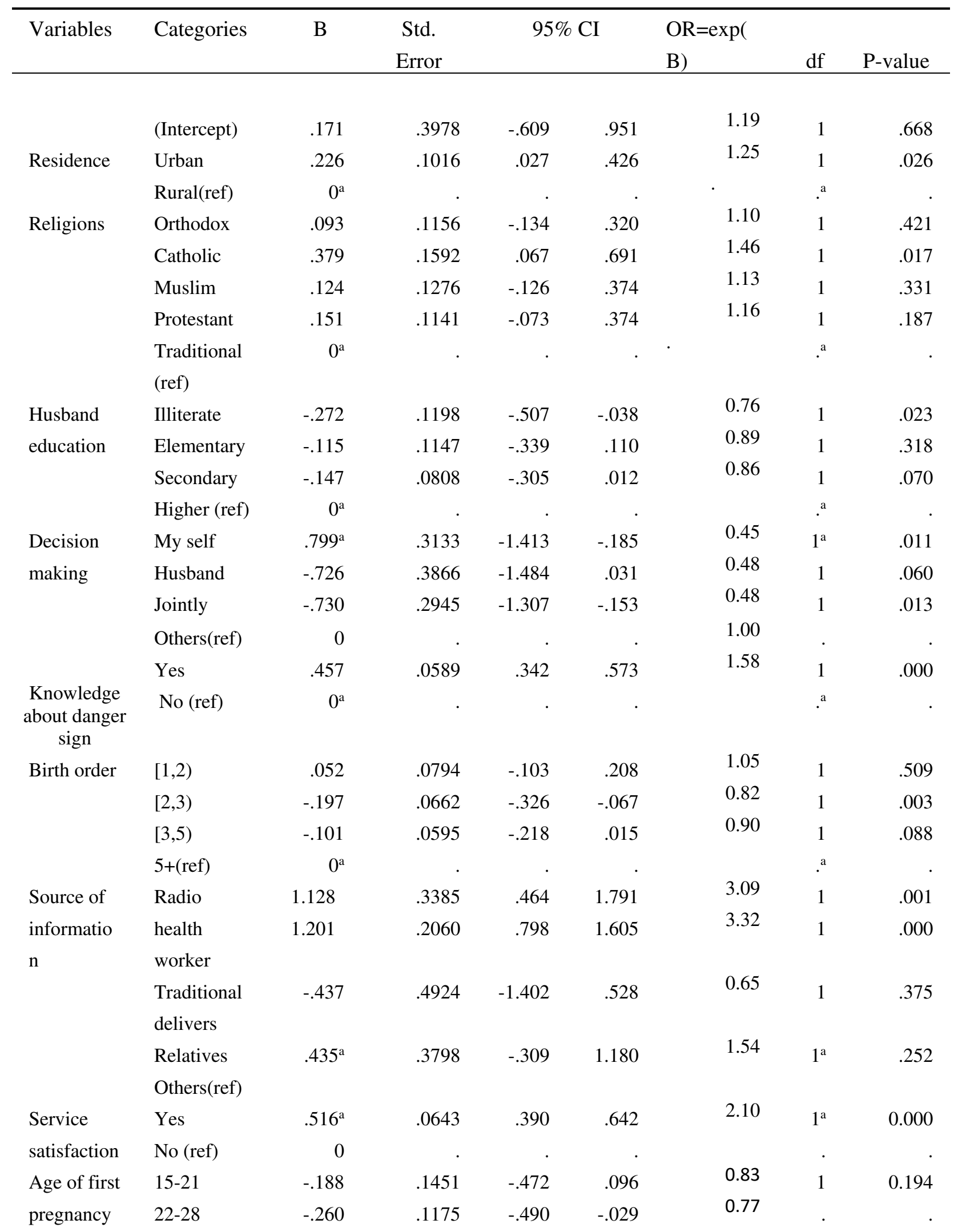




\begin{tabular}{lrrrrrrr}
$29-35$ & $-.162^{\mathrm{a}}$ & .1126 & -.382 & .059 & 0.85 & 1 & 0.195 \\
$36-42$ & -.140 & .0918 & -.320 & .040 & 0.87 & 1 & 0.027 \\
$43-49$ (ref) & 0 &. &. &. & & $1^{\mathrm{a}}$ & \\
\hline
\end{tabular}

Household decision making, knowledge of women on danger sign of pregnancy complication, gain information, service satisfaction, and age of women at early pregnancy are significantly influence the women on antenatal care visits while they are pregnant. From the output given in table 4, the women who decided her self has an odds ratio equal to $\exp (0.799)=0.45$ (95\% CI:$1.413,-0.185)$, indicates that the women who had decided her self are 0.45 less likely to visit than women who had decided by other concerned bodies keeping other variables are constant and similarly women who decided jointly had an odds ratio equal to $\exp (-0.730)=0.48(95 \% \mathrm{CI}$ :$1.307,-0.153)$. It indicated that the women who decided jointly were 0.48 times less likely to attend in the health institution than women decided with others. Source of information such as radio and health workers and its odds ratio respectively is equal to $\exp ($ $1.12)=3.09(95 \% \mathrm{CI}: 0.464,1.791)$ and $\exp (1.20)=3.32(95 \%(0.798,1.605)$ implies that the women who got information from radio and health workers respectively are 3.09 and3.32 times more likely to visit then women got from other information.

\section{Discussion}

The current study aimed to judge factors associated with the ANC visits of women in the BenchiSheko zone. Using the primary source of data which has been collected from different kebeles, seven hundred sixty-four childbearing mothers who had to give birth within five years priorly, 83.1 percent of women attended ANC visits in health institutions at least once [17]. The current study supported by the study done [18], how ever, the current study is some how small in percentage of number of visit. Around seventeen percent of women did not utilize ANC visit, forty-four percent of women attended 4 times and bellow a minimum of WHO recommendation. Around thirty-six women have not utilized according to WHO guidelines.

The study showed that the age of women who had attended ANC visits varied from youngest to oldest. Women at the age of 15 , the number of ANC visit is low. Whereas women at the age of 29 to 35 somewhat increase and women at the oldest age are extremely decreased. This might be the fertility rate may differ within age differences. The current study was consistent with the study done [4], [19]. 
The result showed that child-bearing mothers who had known danger signs of pregnancy complications greater than as compared to women who did not know it. This might be due to the traditional mechanism and maybe health service access to the Bench-Sheko zone. This study was consistent with other studies done [3]. The education of women in the study showed that the number of ANC visit varies. Few women recorded that the women were not educated. It is noted that the number of ANC visits increases as per upgraded their education status. This is suggested that awareness comes from education and it is important. This study much related to the study done somewhere[20], [10].

From Women, those who had lived in rural residents were less likely to have ANC visits. According to the current study the women who had lived in urban had an odds ratio of 1.25(95\%CI:0.27,0.426). It implies that women who had lived in rural residents attended less number of ANC visits. This may be a lack of clinics or health institutions. This study is supported by the study have done [21]-[23].

The study showed that the age of mother at early pregnancy was significantly influencing the utilization of antenatal care visits even if the attendance of antenatal care visits with age varies. Because the ANC visits of child-bearing mothers in the oldest age is likely to have ANC visit than the youngest one. This study is supported by the study on the number of antenatal care done [9], [24], [6].

Based on the final model, birth order, decision making, residence, and education status are significant variables that influence the ANC visits of women. Thus, women whose husband education status is literate are more likely to utilize ANC visit as compared to mothers' husband education status is illiterate, women's birth order or birth interval between 2 and 3 are more likely to attend ANC visit than whose birth interval above 5, women who decided herself or jointly are more likely to have ANC visit as compared to those who are decided by others and the women who lived in rural residents were less likely to have ANC visit as compared to those who had lived in urban resident [25].

\section{Strength and Limitation}

This study is very important that used to assess the socio-demographic factors on ANC visit of childbearing mothers in the Benchi-Sheko Zone, SNNPR, Ethiopia using a representative model. 
In many studies, the study was done using the binary logistic regression model to account for the data by deauthorizing the number of visits, however, it is confusing to say women utilize ANC visits during pregnancy. There fore, the stud tells us how many times the women attend ANC during pregnancy by counting principles. The only limitation of the study that there is no intervention and recalling bias during the survey of the data.

\section{Conclusions}

The current study revealed that residence of child-bearing mothers, source of information obtained, birth order, husband education status, knowledge of mothers about danger sign of pregnancy, age at first pregnancy, service satisfaction, religion and decision making are significantly influencing on the number of ANC visit on women during pregnancy. By educating the households, creating awareness about the danger sign of pregnancy, accessing the source of information, and increasing service satisfaction, it is possible to reduce the deaths of women and babies from pregnancy complications.

\section{List abbreviations}

AIC: Akkakian Information Criteria

EDHS: Ethiopia Demographic and Health Survey

OR: Odds ratio

SNNPR: South Nation Nationalities Peoples of Region

\section{Declarations}

\section{Ethics approval and consent to participate}

The study was approved by the research and community service standing committee of the Mizan-Tepi University, and the permission letter with reference number MTU/02/2017 was confirmed.

\section{Consent for publication}

Not applicable

\section{Competing Interests}

No potential conflicts of interest are declared

\section{Availability of data and material}

The data will be given by the corresponding author upon request.

\section{Funding}


The funding was sponsored by Mizan-Tepi University regardless of designing research methodology, analysis and manuscript preparation.

\section{Authors' contributions}

Assaye Belay Gelaw was responsible for designing the methodology of the research, analyzing, interpreting, and drafting the manuscript.

Abiyot Negash Terefe was responsible for validating the research question, designing, analyzing, and interpret the data.

Solomon Abebaw was responsible for analysis and writing the manuscript.

\section{Acknowledgments}

The data collectors, academic staff of the University, and the research officers are duly acknowledged.

\section{Reference}

[1] M. B. Geta and W. W. Yallew, "Early Initiation of Antenatal Care and Factors Associated with Early Antenatal Care Initiation at Health Facilities in Southern Ethiopia," Adv. Public Heal., vol. 2017, pp. 1-6, 2017, doi: 10.1155/2017/1624245.

[2] T. Tekelab, C. Chojenta, R. Smith, and D. Loxton, "Factors affecting utilization of antenatal care in Ethiopia: A systematic review and metaanalysis," PLoS One, vol. 14, no. 4, pp. 1-24, 2019, doi: 10.1371/journal.pone.0214848.

[3] G. G. Weldearegawi, B. F. Teklehaimanot, H. T. Gebru, Z. A. Gebrezgi, K. B. Tekola, and M. F. Baraki, "Determinants of late antenatal care follow up among pregnant women in Easter zone Tigray, Northern Ethiopia, 2018: unmatched case-control study," BMC Res. Notes, vol. 12, no. 1, pp. 1-9, 2019, doi: 10.1186/s13104-019-4789-8.

[4] T. K. Tegegne, C. Chojenta, T. Getachew, R. Smith, and D. Loxton, "Antenatal care use in Ethiopia: A spatial and multilevel analysis," BMC Pregnancy Childbirth, vol. 19, no. 1, p. V, 2019, doi: 10.1186/s12884-019-2550-x.

[5] C. A. Moyer, P. Dako-Gyeke, and R. M. Adanu, "Facility-based delivery and maternal and early neonatal mortality in sub-Saharan Africa: a regional review of the literature.," Afr. J. Reprod. Health, vol. 17, no. 3, pp. 30-43, 2013.

[6] T. W. Gudayu, S. M. Woldeyohannes, and A. A. Abdo, "Timing and factors associated with first antenatal care booking among pregnant mothers in Gondar Town; North West Ethiopia," BMC Pregnancy Childbirth, vol. 14, no. 1, 2014, doi: 10.1186/1471-2393-14287.

[7] T. Belayneh, M. Adefris, and G. Andargie, "Previous early antenatal service utilization improves timely booking: Cross-sectional study at university of Gondar Hospital, northwest Ethiopia," J. Pregnancy, vol. 2014, no. July, 2014, doi: 10.1155/2014/132494.

[8] EDHS, FEDERAL DEMOCRATIC REPUBLIC OF ETHIOPIA Demographic and Health Survey. 2016.

[9] A. N. Terefe and A. B. Gelaw, "Determinants of Antenatal Care Visit Utilization of ChildBearing Mothers in Kaffa, Sheka, and Bench Maji Zones of SNNPR, Southwestern Ethiopia," Heal. Serv. Res. Manag. Epidemiol., vol. 6, pp. 1-11, 2019, doi: 
$10.1177 / 2333392819866620$.

[10] J. Ebonwu, A. Mumbauer, M. Uys, M. L. Wainberg, and A. Medina-Marino, "Determinants of late antenatal care presentation in rural and peri-urban communities in South Africa: A cross-sectional study," PLoS One, vol. 13, no. 3, pp. 1-16, 2018, doi: 10.1371/journal.pone.0191903.

[11] T. Wondimu and F. Gebresenbet, "Resourcing land, dynamics of exclusion and conflict in the Maji area, Ethiopia," Conflict, Secur. Dev., vol. 18, no. 6, pp. 547-570, 2018, doi: 10.1080/14678802.2018.1532644.

[12] D. Koletsi and N. Pandis, "Poisson regression," Am. J. Orthod. Dentofac. Orthop., vol. 152, no. 2, pp. 284-285, 2017, doi: 10.1016/j.ajodo.2017.05.009.

[13] A. C. Cameron and F. A. G. Windmeijer, "R-Squared Measures for Count Data Regression Models with Applications to Health-Care Utilization," J. Bus. Econ. Stat., vol. 14, no. 2, p. 209, 1996, doi: 10.2307/1392433.

[14] A. N. Sharma, K. M. Elased, and J. B. Lucot, "Rosiglitazone treatment reversed depression- but not psychosis-like behavior of db/db diabetic mice," J. Psychopharmacol., vol. 26, no. 5, pp. 724-732, 2012, doi: 10.1177/0269881111434620.

[15] M. Allen, "Maximum Likelihood Estimation," SAGE Encycl. Commun. Res. Methods, pp. 1-7, 2017, doi: 10.4135/9781483381411.n322.

[16] J. I. Myung, Y. Tang, and M. A. Pitt, "Chapter 11 Evaluation and Comparison of Computational Models," Methods Enzymol., vol. 454, no. C, pp. 287-304, 2009, doi: 10.1016/S0076-6879(08)03811-1.

[17] D. Dulla, D. Daka, and N. Wakgari, "Antenatal Care Utilization and Its Associated Factors among Pregnant Women in Boricha District, Southern Ethiopia," Divers. Equal. Heal. Care, vol. 14, no. 2, pp. 76-84, 2017, doi: 10.21767/2049-5471.100096.

[18] M. Harris and M. Harris, "The New Zealand Project," New Zeal. Proj., no. July, 2017, doi: 10.7810/9780947492588_1.

[19] Z. Abosse, M. Woldie, and S. Ololo, "Factors Influencing Antenatal Care Service Utilization in Hadiya Zone," Ethiop. J. Health Sci., vol. 20, no. 2, 2011, doi: 10.4314/ejhs.v20i2.69432.

[20] S. Pandey, "Socio-economic and Demographic Determinants of Antenatal Care Services Utilization in Central Nepal," Int. J. MCH AIDS, vol. 2, no. 2, pp. 212-219, 2013, doi: 10.21106/ijma.27.

[21] S. Atuhaire and J. F. Mugisha, "Determinants of antenatal care visits and their impact on the choice of birthplace among mothers in Uganda : a systematic review," vol. 11, no. 1, pp. 77-81, 2020, doi: 10.15406/ogij.2020.11.00492.

[22] G. W. Basha, "Factors Affecting the Utilization of a Minimum of Four Antenatal Care Services in Ethiopia," Obstet. Gynecol. Int., vol. 2019, 2019, doi: 10.1155/2019/5036783.

[23] S. K. Ousman, I. Mdala, V. C. Thorsen, J. Sundby, and J. H. Magnus, "Social determinants of antenatal care service use in ethiopia: Changes over a 15-year span," Front. Public Heal., vol. 7, no. JUN, pp. 1-10, 2019, doi: 10.3389/fpubh.2019.00161.

[24] Central Statistical Agency Ethiopia and MEASURE DHS - ICF Macro, "Ethiopia Demographic and Health Survey 2011: Preliminary Report,” pp. 1-29, 2011.

[25] M. W. Azimi et al., "Factors associated with antenatal care visits in Afghanistan: Secondary analysis of Afghanistan Demographic and Health Survey 2015," Nagoya J. Med. Sci., vol. 81, no. 1, pp. 121-131, 2019, doi: 10.18999/nagjms.81.1.121. 